\title{
The role of operational research in clinical chemistry
}

\author{
J. PETO ${ }^{1}$, L. G. WHITBY, AND D. J. FINNEY \\ From the Departments of Statistics and Clinical Chemistry, University of Edinburgh
}

SYNOPSIS This paper begins with a critical examination of the published account (Carruthers, 1970) of a pioneering study of simulation in a clinical chemistry laboratory. It next considers the basic requirements for the formal mathematical description of such a laboratory: these include the setting of criteria, the resource and input variates, and the definition of success by means of criterion variates. Investigation of criterion variates can be by direct measurement, or by a theoretical approach which may include simulation. The aims of simulation are discussed and an attempt to simulate the operation of a large clinical chemistry laboratory is described.

The difficulty of constructing an overall measure of efficiency is considered in relation to improving performance within an existing framework, to evaluating new equipment such as computers on-line to laboratory apparatus, and to inter-laboratory comparisons of performance. It is concluded that complex operational research techniques, including simulation, have little to offer at least for the present, and may even lead to misleading conclusions.

Clinical chemistry laboratories are experiencing rapid increases in the number of analyses performed, often as much as a doubling every four years. To cope with these work loads, automatic methods of analysis have been introduced on a large scale, and these have helped to overcome shortages of trained staff. Increasing work loads, shortages of staff, and the efficient operation of expensive automatic equipment demand good organization and operation of the laboratory. In most big laboratories these features, and particularly the automatic equipment, have created a factory situation for a large proportion of the specimens handled: in principle, a sample requiring a specified set of assays passes, or should pass, through the various parts of a laboratory in a fixed order and on a fixed schedule. Factory methods of operation naturally suggest that operational research techniques, which have proved so useful in industrial planning and reorganization, might assist the study of laboratory efficiency.

The operational research approach to a problem normally involves definition of an overall index, of efficiency or profit, maximization of which is asserted to determine the best mode of operation. In principle, this is the ideal basis for decision. In practice, however, establishment of a realistic index may be so

${ }^{2}$ Present address: Institute of Psychiatry, University of London.

Requests for reprints to Professor L. G. Whitby, Department of Clinical Chemistry, University of Edinburgh.

Received for publication 21 August 1972. complex that recourse is had to arbitrary estimates of the financial consequences of possible changes. A medical environment presents peculiar difficulties in obtaining agreed values, on a monetary or any other scale, to be placed on benefits to patient care, improvements in the use of time by physicians, and increases in hospital efficiency. Two main types of operational research can be distinguished:

\section{Direct Measurement}

In this, the introduction of new equipment or new organization is compared with the situation previously obtaining.

\section{Theory}

This attempts to predict the effects of possible but as yet untried changes in organization and equipment.

Direct measurement has the attraction of seeming to be realistic, but its application is limited to circumstances where advantage can be taken of changes already approved, or so minor as to be acceptable experimentally for a short period. Moreover, since any major change in equipment, eg, the introduction of a laboratory computer, is likely to be accompanied by changes in staffing dispositions and organization not in themselves essential to the change in equipment, but made contemporaneously for a 
variety of reasons, discrimination between effects may be impossible.

Theoretical studies may range from simple arithmetic evaluation of the extra work that might be undertaken if an additional technician is employed (assuming an average and predictable work content), through mathematical analysis of rates of flow of work, to the numerical simulation on a computer programmed to correspond to the whole performance of a system. Although the theoretical approach avoids many of the problems of direct measurement, it is often open to the criticism that the theory fails to represent realities completely and exactly.

Operational research methods have been applied to the study of outpatient departments (BlancoWhite and Pike, 1964), surgical admissions (Weir, Fowler, and Dingwall-Fordyce, 1968), $x$-ray departments (Fraser, 1969; Jeans, Berger, and Gill, 1972), haematology (Rath, Alverez Balbas, Ikeda, and Kennedy, 1970), and clinical chemistry laboratories (Carruthers, 1970). Examples of relatively simple but arbitrary indices of efficiency or profit have included attempts to apply a costing figure to alterations in the time required for reporting results of laboratory work, and to place monetary values on work conducted at different levels of analytical precision. The purpose of this paper is to discuss the application of operational research techniques to clinical chemistry laboratories.

Simulation in a Clinical Chemistry Laboratory: a Discussion of the Example Reported by Carruthers (1970)

Critical examination of this pioneering study draws attention to some of the main issues facing any user of operational research in a large clinical chemistry laboratory, and helps to identify some of the principles on which further advances must be based. The author described three laboratory schedules, listing for each assay and each day the latest time that a specimen could be accepted for analysis on that day. For each schedule, the delay suffered by each of a random sample of request forms before all the analyses requested had been completed was found by a computer simulation. The financial consequences of the different schedules were estimated on the assumption that each day in hospital cost the community at least $£ 10$, and that at least $10 \%$ of the delays in the issue of reports from the laboratory attributable to the slower schedules of work resulted in consequential delays in the discharge of inpatients from hospital. Carruthers suggested that comparison of these estimated financial consequences with the costs of implementing the different laboratory schedules would identify the best schedule. Certain assumptions implicit in this method must be 0 examined.

In the first place, the capacities of the several systems were assumed to be adequate and therefore $\vec{\Rightarrow}$ irrelevant to the selection of the best schedule. In $\stackrel{5}{+}$ practice, there is no simple relationship between work load and a laboratory's theoretical analytical 음 capacity since automatic equipment may be installed $\frac{\sigma}{\sigma}$

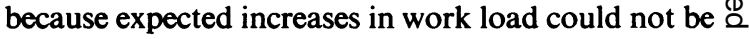
accommodated and standards of reliability maintained by non-automated methods (eg, Whitby, $\vec{O}$ 1967). On the other hand, it is not always reasonable in practice to ignore the effects of technical break- $\vec{\omega}$ downs on analytical capacity, as mentioned again later.

Secondly, in comparing the cost-effectiveness of is the three schedules, Carruthers assumed, for an arbitrary proportion of cases, that delay in reporting $\overrightarrow{0}$ results prolonged the stay of patients in hospital by an amount corresponding to the difference in 을 reporting times attainable with the different schedules. He appears to have assumed, tacitly, that all $z$ results corresponding to a particular request would $\stackrel{\succ}{\succ}$ be reported together. In fact, it is widely held $\frac{\Phi}{3}$ laboratory practice to report results on the day they become available (as interim reports) rather than wait until all assays requested on a specimen have $\overrightarrow{0}$ been completed. Furthermore, clinical decision $N$ about treatment are only sometimes held up until the results of chemical investigations become available and it is unusual for patients who are clinically fit for discharge to have the time of their discharge delayed pending the results of chemical investigations. It is questionable, therefore, whether delays in reporting results would have direct and consequential effects on the time of discharge on the scale suggested by Carruthers, even assuming that all the request forms he was reviewing had originated from inpatients. If some of the request forms had come from outpatients, delays in the laboratory would probably not have had significant consequent:al effects on the costs to the National Health Service for these analyses; in evaluating any schedule it is important to know the sources from which requests are drawn as well as the types of analyses being requested.

A third assumption by Carruthers was that variation in the rate of arrival of requests for analysis, $N$ not sufficient to produce persistent queues, was the only stochastic element in the laboratory: by $\mathcal{N}$ 'stochastic' is meant 'dependent on effectively $\tilde{\omega}$ random chances and expressible only in terms of $\underset{2}{\sigma}$ probabilities instead of being exactly calculable'. 0 Although this simplification would be acceptable if $\stackrel{\odot}{\Phi}$ the work load were large enough to be stable and the $\stackrel{\odot}{+}$ analytical equipment reasonably reliable, in everyday $T$ work some requesting rates show considerable 
variation and failures in machine performance can cause substantial delays in starting or completing analyses.

These criticisms mean that this simulation study failed to take account of the fact that the financial evaluation of any laboratory schedule of work should be based on a detailed knowledge of the sources and types of specimen affected, as well as the distribution of their arrival times in the laboratory throughout the day. Important though these criticisms are on points of detail, they do not detract from the assertion by Carruthers that simple and useful specification of laboratory schedules is possible, nor from his recognition that delays in performing laboratory work can have direct-although not easily assessedfinancial consequences. Nevertheless, the criticisms must be taken as a warning against the temptation to exaggerate the accuracy of apparently sophisticated methods, and to claim that they therefore lead automatically to the best decision.

Another drawback to this approach is that it is unlikely to lead to the optimal allocation of resources, since only complete work schedules are being considered. For example, changing from the first to the second schedule described by Carruthers (1970) would, amongst other effects, reduce the maximum delay in the determination of serum protein-bound iodine (PBI) from one week to three days. If this change could be effected quite cheaply for PBI determinations alone, it might be well worth while, but it need not require changing the laboratory's whole method of operation to schedule 2 . Simple inspection of data relating to specific assays or groups of assays, as tabulated by Carruthers, shows where the greatest improvements in laboratory performance can be obtained from changing work schedules; inspection by itself is likely to lead towards the best schedule, providing data about sources of specimens and their arrival times in the laboratory are available.

\section{The Formal Mathematical Description of an Analytical Laboratory}

A fundamental reason for studying the performance of a hospital laboratory, or system of laboratories, is to find ways of improving performance. Such studies usually start from the assumption that the work is being done well-for instance, that every chemical analysis has acceptable standards of reliability.

Any processing system, whether a factory or an analytical laboratory, consists of a set of elementary units, each of which has its own characteristics of operation; the units may be arranged in series, in parallel, or in a combination of the two. If the performance of each unit can be expressed quanti- tatively in respect of the time it requires to fulfil its function, or of constraints on the quality and quantity of its performance, together with any necessary stochastic element to represent the inherent variability of each unit, theoretically the performance of the whole system can be predicted. Besides variability within the system, stochastic variation in the input may require specification. Unless all stochastic variation is negligible, the output of a processing system will be subject to random variation; description of this is often of fundamental importance for the evaluation of the system. In simple systems, the structure may allow the equations relating output to input, and to the behaviour of the elementary units, to be solved completely; quantitative evaluation of any property of the system can be achieved. The system does not have to be very complicated, however, before a mathematical solution of the equations becomes impossible.

\section{SETTING OF CRITERIA}

Interest lies in the effects of changing the many factors that define the facilities of the laboratory and their manner of utilization. These include numbers of staff in various categories, numbers and types of items of equipment (AutoAnalyzers ${ }^{1}$, etc), and modes of operation, sizes of 'stores' (samples awaiting analysis, patients awaiting tests, etc) and scheduling of the programme of work in relation to different tests. These factors can all be expressed quantitatively, although adequate description of such concepts as scheduling possibilities is complicated. The factors constitute an array of different variates, for instance: $x_{1}=$ number of AutoAnalyzers; $x_{2}=$ number of technicians; $x_{3}=$ time of day for last acceptance of samples for test A, etc. These factors may be collectively described as the 'resource variates' and collectively referred to as $x$ without a subscript.

A second category of variates, $y$, is needed to define the input. These specify the daily demands on the laboratory (numbers of samples classified according to analyses requested, times of receipt of samples, etc). They may be named $y_{1}, y_{2} \ldots$ and termed the 'input variates'. Resource variates are within the control of the laboratory management, apart from limitations of finance and staff recruitment, whereas input variates correspond to external demands.

A third category of variates is needed to define success, the 'criterion variates'. Although ways of improving he performance of the laboratory are being sought, the proper choice of a measure of success is not self-evident; the number of samples analyzed per day, the time from receipt of a sample ${ }^{1}$ Technicon Instruments Co., Basingstoke, Hants. 
to reporting the corresponding analyses, the percentage of samples not analysed and reported completely within 24 hours, etc, are all possibilities. These different criterion variates, named $z_{1}, z_{2} \ldots$ (collectively referred to as $z$ ) may be in conflict. For example, other things being equal, a scheduling change designed to reduce the time for processing a particularly important sample may reduce the total number of samples analysed that day.

\section{MEASUREMENT OF CRITERIA}

Recommendations for improving laboratory performance can be derived only if the relevant criterion variates $(z)$ are to some extent dependent upon the resource $(x)$ and input $(y)$ variates chosen for measurement, and if, for stated values of the $x$ and $y$ variates, prediction of one or more of the $z$ variates is practicable. Exact determination of $z_{2}$, for instance, cannot be expected from knowledge of the $x$ and $y$ variates because the measured $x$ variates are at best only the more important members of a much larger set.

For purposes of inference, any criterion variate needs to be averaged over the various conditions represented by the frequency distribution of the input variates, since normally the latter cannot be controlled by the laboratory. Unless the variability arising from stochastic sources and from unmeasured resource variates is negligible, for a criterion such as the time required to process a sample the distribution of the variate about its mean value will also need to be taken into account. The mean and standard deviation of the time per sample might then be adopted as two distinct criterion variates, since overall assessment of the quality of laboratory performance must recognize that excessive variability may seriously reduce the acceptability of a particular work schedule, no matter how satisfactory the average processing time may seem. If the frequency distribution of a $z$ variate for specified values of $x, y$ is very skew, percentiles or probabilities may be more useful. For example, the processing time exceeded by the most extreme $10 \%$ of samples, or the probability that the analysis of a sample will not be completed within 48 hours, may be useful measures of efficiency.

Values for $z$ variates corresponding to specified values of $x, y$ may be sought either by direct measurement or by theory. If normal laboratory operation permits $z_{3}$, for instance, to be recorded on a number of occasions where different $x, y$ either occur naturally or can be adjusted to suit the investigation, empirical knowledge of the relation between $z_{3}$ and $x, y$ can be built up. Provided that the relation is fairly regular and variability is not too great, interpolation may then allow adequate prediction of $z_{3}$ for other combinations of $x, y$ values not previously tried. However, if the number of $x$ and $y$ variates is large, and if determination of a single value of $z_{3}$ requires the records for a whole day's laboratory work, accumulation of sufficient data will take a long time. In addition, the effects of irrelevant changes may be included within the measured criteria. Perhaps the biggest disadvantage, however, is the fact that the ranges of values of resource variates that can be studied, in the course of normal operation, will commonly be much narrower than are of interest for inferring the optimal structure.

The alternative, theoretical approach covers a much wider range of possibilities. In the simplest systems, elementary arithmetic may suffice to connect $z_{3}$ with $x, y$ as in a physical system in which neither human intervention nor occasional faults disturb the uniformity. If a liquid is to be conveyed by a straight pipe, for example, the volume carried will depend on the diameter of the pipe and on the pressure and viscosity of the liquid. Even if $z_{3}$ is not exactly determined by $x, y$, the system may permit maximum and minimum values to be calculated by extreme assumptions. However, full theoretical specification of a complex system often leads to sets of equations that are intractable by formal mathematical analysis. It is important also to remember that the theoretical approach is valid only in so far as the equations used adequately describe the beha viour of the system for all combinations of $x, y$ variates and this holds whether the equations are solve mathematically or subjected to simulation.

SIMULATION AS APPLIED TO AN ANALYTICAL L A BOR A T OR Y

Simulation is often used as an alternative to the theoretical formal description of a process. It is usually assumed to refer to a computer technique, but this is not a necessary feature. In simulation, the history of a single product or sample passing through the system is followed according to the rules applicable to each unit, every instance of stochastic variation being taken into account by a random selection from the appropriate frequency distribution. In this way, the final properties of one particular simulated item running through the whole process can be described numerically. Repetition of these calculations, with a new random selection of variables each time, enables frequency distributions to be constructed for the properties of the output from the system. If different versions of the whole system are to be compared, each can be simulated sufficiently often to ensure that any important differences are made apparent by the forms of the final frequency distributions.

Expressed in these terms, simulation may sound very attractive, but it is appropriate only when the 
system to be analyzed is too complex for the consequences of the alternative systems under consideration to be calculated by a direct mathematical approach. Simulation can have one of three aims: (1) to predict the effects of changes in the system, or in the input to the system, ie, changes in $x$ and $y$ variates, upon the operation and upon particular criterion $(z)$ variates; (2) provided that the effects just mentioned can be evaluated financially, or combined into a single index of efficiency, to generate numerical values of the index for different $x, y ;(3)$ to maximize this index of efficiency, and thereby to find automatically the optimal conditions of operation. Generally, the third aim is only practicable when a computer is used for the simulation, with appropriate programming to vary the simulated structure so as to make it evolve towards the optimal conditions. Prerequisites for the employment of simulation are:

1 Enough is known about the manner in which $z$ depends upon $x, y$ for the simulation to be constructed realistically, even though the corresponding equations may defy explicit solution.

2 The components of random variation introduced into the simulation or permitted to occur are quantitatively good approximations to those of the real system.

3 The ranges of $x$ and $y$ that can be covered by the simulation are at least as wide as those appropriate to the real system.

Unless these prerequisites are met, there is a definite danger that the simulation will correspond less to reality than to the investigator's concept of how the system should perform. Thus simulation must assume very close (ideally, exact) correspondence between simulated values of $z_{1}$ and values that would be observed for the same $x, y$. Also, knowledge of the theory must suffice for interpolation within the framework of the simulation; simulation measures $z_{1}$ for specified $x, y$ but subsequent inference may require to estimate $z_{1}$ for intermediate $x, y$.

Investigation of rules that enable criteria to be predicted from $x, y$ may demand recourse to simulation if some of the following conditions obtain:

$a$ The formulae relating a criterion variate to $x, y$ are so complicated that the theory is intractable and accumulation of sufficient observations from the real system would take too long a time.

$b$ Records from an observational approach would be acquired very slowly (eg, one per day), whereas simulation makes possible a much more rapid advance in understanding:

$c$ For specified values of $x$ and $y$, variation in $z_{1}$ is so great (on account of unidentified or unmeasurable variates) that large numbers of observations would be required even to estimate the mean of $z_{1}$, and still larger numbers for any study of the frequency distribution of individual values about the mean.

$d$ The extent to which resource and input variates can be varied, or will vary naturally while observations are being collected, is much less than the range desired for future inference.

$e$ In the particular circumstances, simulation is substantially cheaper than feasible alternatives.

\section{Further Observations Relevant to Operational Re- search in Clinical Chemistry}

The concepts discussed in this paper are relevant to a study which began with the collection of data relating to the clinical chemistry laboratory in the Royal Infirmary, Edinburgh, on the basis of which the intention was to develop a simulation program or a mathematical model. The flow of work through such a laboratory is basically simple. Specimens arrive at the reception area and samples are distributed to the appropriate analytical benches where much of the work is carried out with AutoAnalyzers. The raw data are recorded, corrected, and converted to the relevant units for reporting purposes, and issued as reports after collation and assessment. From the analytical viewpoint, if the equipment is reliable, each bench should be capable of operating at a predictable speed throughout the day except for periods of maintenance of equipment and preparation for analysis. Any tendency for occasional overloading in the reception area tends to be met by the temporary redeployment of staff; regular overloading would require more staff or could theoretically be catered for by alternative arrangements such as staggering the times for submission of requests from different wards or clinicians.

As indicated, the initial approach was by simulation. Distributions of arrival times of specimens requiring particular assays or groups of assays were observed, as were the normal starting times on each bench, time spent in the reception area while specimens were registered and prepared for analysis before distribution to the appropriate bench or benches, and so on. The simulation program could allow for the progressively reduced service which would operate if increasing proportions of the staff were absent.

The first prerequisite of any simulation must be that, when it is based on the observed distributions of $x$ and $y$, the resulting distributions of the criterion variates, $z$, are very similar to those currently achieved. The initial program failed to do this-the simulation described the 'official' timetable, corresponding very closely to the schedule achieved on the few days when no interruptions occurred. Allowance 
was then made for the observed distributions of various delays, including equipment failure, the rerunning of unsatisfactory specimens, delays in changing from one analysis to another, etc, thereby producing a simulation which was virtually indistinguishable, at least in output, from the laboratory in operation under the then existing conditions.

The question next arose whether it was possible or desirable to proceed to simulate alternative arrangements, assuming that the delays now incorporated into the program were inherent properties of the system. It was clear that the optimal capacity of the laboratory was equal to the number of specimens that the staff and equipment could analyse if they worked continuously from 9 am to $5 \mathrm{pm}$; such a schedule would require specimens to arrive early enough for AutoAnalyzers to operate without interruption, and possibly extra staff in the reception area, but these requirements could have been met theoretically. However, it was clear that a computer was hardly necessary to calculate the corresponding capacity, which was the theoretical optimum for the laboratory. More marginal improvements could readily be predicted without recourse to the computer if assays were to be grouped differently, or the way in which specimens were distributed modified, without altering the existing operating characteristics of the separate units in the simulation. In the belief that the computer simulation was unnecessarily sophisticated, the actual processes in the laboratory were next examined further.

With the exception of automatic equipment used for assaying different substances on the same day, analysis rates and the length of time during which automatic equipment was operated should have determined the capacity of the laboratory for those assays carried out on this type of apparatus. Applying such simple calculations, it soon became apparent that the automatic equipment, mostly Mark I AutoAnalyzers, could (with the exception of the urea and electrolyte analyzer) theoretically handle about twice the current demand for analyses. The difference between the theoretical and actual throughputs of work, in those instances where queues built up, was largely attributable to machine failures of varying duration and grades of severity. The scheduling of usage for most of the automatic equipment was, however, deliberately being kept sufficiently lax as to leave a reasonable margin for dealing with these unscheduled breakdowns; laxity of scheduling is clearly necessary if machine failure is common. If throughput were to be increased and maintained without additional capital expenditure, and the efficiency of use of staff and equipment thereby improved, observations on each assay over a period were needed rather than theoretically sophisticated operational research (whether by simulation or otherwise) to determine the amounts of potential operating time lost.

These observations were made and were divided between machine failures per se and other losses (late starting for reasons other than machine failure, delayed changing of plates of specimens for AutoAnalyzers, time spent on analyzing duplicate samples, etc), and the causes of loss of efficiency recorded. Since AutoAnalyzers run at constant speeds, widely spaced visits to each bench sufficed for recording the starting and stopping times for analyses and for identifying the occurrence of delays. Discrepancies between theoretical and actual throughputs of specimens were then clarified by enquiries made the same day from the staff concerned. Some delays were undoubtedly due to human error, but these were sufficiently rare for excessive questioning to be unnecessary and for cooperation not to be impaired by any implication that the competence of staff was the real subject of the study.

After comparing current performance with the best theoretically achievable, given high machine reliability and rigid scheduling, steps could be taken to reduce the larger deficiencies. On the basis of observed performance, improvement in the reliability of AutoAnalyzer equipment seemed to be the chieB need, particularly of the Technicon Mark III flame photometei, with conditions of operation during the day so organized as to interrupt the continuous flow of analyses as little as possible. To this end, with standard (non-computerized) methods of chart reading, staff duties needed to be arranged so that chart reading and calculation of results corresponding to the preceding plate of samples, and preparation of specimens and loading of the subsequent sample plate, were undertaken, on a long analytical run, during the time that the current plate of samples was being analysed. Improvements in machine reliability, eg, from preventive maintenance, that reduced the down time before the start of a run, or the frequency of breakdowns in the middle of a run, improved efficiency by lessening the time spent on diagnosing faults or on repeating the analysis of specimens adversely affected by machine failure.

\section{Discussion}

Operational research is intended to provide a basis for inference about the consequences of changes in a system, especially changes in the resource variates $(x)$ introduced as a policy for improving performance but also changes in the input variates $(y)$. Changes in $y$ are less immediately controllable, but may be contemplated as a long-term development, for example, because of a proposal to adopt a general 
programme of screening patients by a range of laboratory tests, or because of an increase in the catchment area. If the relation between output criteria $(z)$ and $x, y$ has been determined, the procedure is to use this relation to predict how much the criteria will be altered as $x$ and $y$ change, for instance to find optimal values for some or all of the $x$ variates under certain constraints on the values of $y$ that will be encountered. 'Optimality' is defined as producing the most desirable values for one or more criteria, for example, maximizing the utilization of expensive equipment. However, where aims are not entirely compatible, a formal compromise such as a single index of performance based on various criteria may be misleading, since such indices are liable to be more or less arbitrary unless the criteria on which they are based can be accurately assessed financially. It might, for example, be impossible to find a generally acceptable formula which included precision, speed of issuing reports, and working conditions of staff.

Whether the criteria are studied by direct measurement or by theory (with or without simulation), the validity of inferences depends entirely upon the adequacy of the model. If the theory were correct, deductions from it would be in no doubt; in real life, theory seldom has this exactness. Measurement has the advantage of guarding against flaws in the model, by making certain that the $z$ variates correspond with the $x$ and $y$, thus ensuring reasonable estimation of any criterion from knowledge of values $x$ and $y$, provided that other unspecified variates are unimportant. However, if planned changes in the $x$ and $y$ variates are associated with unavoidable or even unsuspected changes in other relevant variates, inferences and predictions may be seriously misleading. Inference becomes more secure if the theoretical and measurement approaches can be used to reinforce one another. The stochastic elements in a clinical chemistry laboratory are few and simple in contrast to clinics, in which attendance by patients is necessary, and where consequently a complex queue structure is encountered. In the laboratory, the most important factors are those that affect the availability and reliability of resources, notably machine breakdowns, reduced hours of operation, and staff shortages. If the effects of such factors can be predicted approximately by elementary calculation, there is little to be gained by more complex study unless the predicted changes in output variates can be precisely evaluated financially. The contention of this paper is that such financial evaluation cannot be done, and that decisions about the most suitable ways of running an individual laboratory should continue to be made by heuristic assessment of the effects of changes in method of operation on output, rather than by invoking sophisticated theory. The comparative evaluation of different systems of laboratory operation is most usefully achieved by listing the precision of analytical work, the speed of reporting results, the costs of running the laboratory, its work load, and its capacity for expansion-information that is mostly available. These data can be satisfactorily recorded and examined as individual items, not needing to be combined into formulae more complex than a simple overall figure such as the average cost per analysis in a laboratory; even this simple figure may be misleading if used to compare two or more laboratories, because laboratories may differ considerably in their patterns of work.

It is, nevertheless, potentially useful to compare the performance of similar laboratories. This should be possible by using a simple proforma for the collection of data such as those listed in the preceding paragraph, and these might draw attention to shortcomings in individual laboratories; no special operational research teams should be needed. Such comparisons could reveal differences in reliability of equipment, or in operating costs, and might serve to indicate standards of reliability and performance that should be able to be approached in routine practice. The main emphasis in inter-laboratory comparisons so far has been on the quality of analytical work; such comparisons of analytical reliability reveal minimal acceptable standards for the quality of performance, since there is no reason theoretically why laboratories using similar equipment should not achieve closely similar results. Examination of discrepancies should reveal differences in the operation of analytical methods, for instance in the maintenance or operation of the equipment.

Simulation finds its greatest usefulness when applied to complex systems, the output characteristics of which can be summarized easily in terms of efficiency or productivity. In such studies, distinction between structural complexity and functional diversity is essential. In a car factory, for example, the effects of changes in the system are difficult to predict because the overall system is structurally complex; the schedulings of the various processes are so interdependent that simulation would probably be needed to predict the effect on output of any changes. To assess the financial value of a given change in output is simple, however, because the sole purpose of the process is the production of cars. A hospital laboratory, by contrast, presents very different problems: to predict at least approximately the effect of changes in operation, eg, in altering the capacity for work, or in affecting the speed of issuing reports, may be easy, but the evaluation of these effects in financial terms may be extremely difficult. 
These considerations again indicate that simulation is not appropriate for operational research applied to a clinical chemistry laboratory.

These conclusions on the dangers of attempting to synthesize overall measures of laboratory efficiency, on which to base decisions on future planning, apply without modification to the evaluation of radically new systems. Several clinical chemistry laboratories have been operating on-line computer systems of various degrees of complexity, and the health departments are naturally concerned that they be compared with traditional systems on some basis of cost-effectiveness. Such comparisons must not be simplified by attempting to separate computerrelated from other activities, and then comparing some derived index such as staff efficiency in these areas with the 'efficiency' previously obtaining. The effects of introducing a computer into a laboratory are inescapably complex and, apart from observed changes in criteria such as analytical precision, speed, cost, work load, and capacity, a comparative evaluation should take account of improvements which could be effected within the original system by methods not requiring the introduction of the computer.

This project was financed by a grant from the Scottish Home and Health Department, and the authors are very grateful for this support. The detailed work was begun by Dr Brenda J. Fraser and continued by one of us (J.P.). We wish to express our thanks to $\mathrm{Dr}$ Fraser, and to the many members of laboratory staff who assisted with this study, particularly Dr I. W. Percy-Robb and Mr J. Proffitt.

\section{Addendum}

Since this paper was submitted, a further account of a laboratory simulation has been published (Cundy, 1972). This study illustrates the main points put forward in the present paper, and could be subjected to detailed criticism. We do not share the author's optimism about the application of his simulation model to the whole of the clinical laboratory system, and would draw attention to the inability of his present model to match existing conditions.

\section{References}

Blanco-White, M. J., and Pike, M. C. (1964). Appointment systems in out-patient clinics and the effect of patients' unpunctuality. Med. Care, 2, 133-141.

Carruthers, M. E. (1970). Computer analysis of routine pathology work schedules using a simulation programme. J. clin. Path., 23, 269-272.

Cundy, A. D. (1972). A simulation study of the clinical laboratory office. In Spectrum 71: a Conference on Medical Computing, edited by M. E. Abrams, pp. 124-138. Butterworths, London.

Fraser, B. J. (1969). The organisation of a radiology department in a district general hospital. Ph.D Thesis, University of Reading.

Jeans, W. D., Berger, S. R., and Gill, R. (1972). Computer simulation model of an X-ray department. Brit. med. J., 1, 675-679.

Rath, G. J., Alverez Balbas, J. M., Ikeda, T., and Kennedy, O. G尺 (Spring 1970). Simulation of a hematology department. Hitto Serv. Res., 5, 25-35.

Weir, R. D., Fowler, G. B., and Dingwall-Fordyce, I. (1968). Thळ prediction and simulation of surgical admissions. In Computers. in the Service of Medicine, vol. 2, edited by G. McLachlan and R. A. Shegog, pp. 141-154. Oxford University Press, London. Whitby, L. G. (1967). Automation in clinical chemistry: a consideration of cost implications. The Hospital, 63, 89-94. 